\title{
Os impactos biopsicossociais sofridos pela população infantil durante a pandemia do COVID-19
}

\author{
The biopsychosocial impacts suffered by the child population during the COVID-19 pandemic \\ Los impactos biopsicosociales sufridos por la población infantil durante la pandemia COVID-19
}

Recebido: 23/01/2021 | Revisado: 24/01/2021 | Aceito: 19/02/2021 | Publicado: 28/02/2021

\author{
Isadora Maria Gomes Almeida \\ ORCID: https://orcid.org/0000-0002-7933-6812 \\ Universidade Federal de Pernambuco, Brasil \\ E-mail: isadoragomes07@hotmail.com \\ Auvani Antunes da Silva Júnior \\ ORCID: https://orcid.org/0000-0003-4030-8707 \\ Universidade Federal de Pernambuco \\ E-mail: auvaniantunes@gmail.com
}

\begin{abstract}
Resumo
Objetivo: discutir sobre o impacto biopsicossocial causado nas crianças devido às medidas de distanciamento e o isolamento social, impostas com o intuito de diminuir a transmissão do novo coronavírus, já relatados na literatura. Metodologia: realizou-se consultas nos bancos de dados da SCIELO, MEDLINE e PUBMED, utilizando os descritores "children", "social isolation", "psychosocial" e "covid-19", com publicações no período de 2019 a 2020. Ao final das buscas, 15 publicações foram selecionadas para compor o estudo. Resultados e Discussão: Observou-se que durante o período de isolamento e distanciamento sociais, as crianças e os adolescentes ficaram mais propensos a desenvolver estresse crônico e agudo, ansiedade, depressão, distúrbios do sono e do apetite, irritabilidade, medo, insegurança e prejuízo nas interações sociais. Os grupos infantis mais vulneráveis, como os jovens que apresentam TEA e TDAH, também sofreram com as mudanças provocadas pela pandemia, apresentando alterações comportamentais. Conclusão: A população infantil está sujeita a sofrer implicações biopsicossociais com as medidas de isolamento social, sendo importante a criação de medidas que visem auxiliar a saúde mental das crianças e dos adolescentes nesse período conturbado.
\end{abstract}

Palavras-chave: Infecção por coronavírus; Isolamento social; Criança; Impacto psicossocial.

\begin{abstract}
Objective: to discuss the biopsychosocial impact caused on children due to distance measures and social isolation, imposed in order to reduce the transmission of the new coronavirus, already reported in the literature. Methodology: consultations were carried out in the SCIELO, MEDLINE and PUBMED databases, using the descriptors "children", "social isolation", "psychosocial" and "covid-19", with publications from 2019 to 2020 . At the end of the searches, 15 publications were selected to compose the study. Results and Discussion: It was observed that during the period of social isolation and detachment, children and adolescents were more likely to develop chronic and acute stress, anxiety, depression, sleep and appetite disorders, irritability, fear, insecurity and injury in social interactions. The most vulnerable child groups, such as young people with ASD and ADHD, also suffered from the changes caused by the pandemic, with behavioral changes. Conclusion: The child population is subject to suffer biopsychosocial implications with measures of social isolation, being important the creation of measures that aim to help the mental health of children and adolescents in this troubled period.
\end{abstract}

Keywords: Coronavirus infection; Social isolation; Child; Psychosocial impact.

\section{Resumen}

Objetivo: discutir el impacto biopsicosocial causado en los niños por las medidas de distanciamiento y aislamiento social, impuestas con el fin de reducir la transmisión del nuevo coronavirus, ya reportado en la literatura. Metodología: las consultas se realizaron en las bases de datos SCIELO, MEDLINE y PUBMED, utilizando los descriptores "niños", "aislamiento social", "psicosocial" y "covid-19", con publicaciones de 2019 a 2020. Al final de las búsquedas, se seleccionaron 15 publicaciones para componer el estudio. Resultados y Discusión: Se observó que durante el período de aislamiento social y desapego, los niños y adolescentes tenían más probabilidades de desarrollar estrés crónico y agudo, ansiedad, depresión, trastornos del sueño y del apetito, irritabilidad, miedo, inseguridad y lesiones. en las interacciones sociales. Los grupos de niños más vulnerables, como los jóvenes con TEA y TDAH, también sufrieron los cambios provocados por la pandemia, con cambios de comportamiento. Conclusión: La población infantil está sujeta 
a sufrir implicaciones biopsicosociales con medidas de aislamiento social, siendo importante la creación de medidas que tengan como objetivo ayudar a la salud mental de niños y adolescentes en este período convulso.

Palabras clave: Infección por coronavirus; Aislamiento social; Niño; Impacto psicosocial.

\section{Introdução}

Atualmente, a população mundial vem enfrentando uma pandemia provocada pelo novo coronavírus denominado de SARS-CoV-2, esse é o vírus responsável pela doença infecciosa conhecida como COVID-19 (Freitas, Alves \& Gaíva, 2020). O acometimento global provocado por essa patologia deve-se a sua característica de alta contagiosidade, uma vez que sua transmissão ocorre facilmente por gotículas respiratórias ou por contato direto, facilitando a multiplicação de pessoas infectadas pelo vírus (Pereira, et al., 2020).

Nessa perspectiva, os dados epidemiológicos evidenciam que esse vírus já infectou mais de dezessete milhões de pessoas e levou a óbito mais setecentas mil pessoas pelo globo, com suas altas taxas de transmissibilidade e letalidade, causando assim uma forte preocupação pelos órgãos de saúde (Brasil, 2020), a COVID-19 é considerada um problema de saúde pública, que ainda não apresenta nenhum tratamento efetivo e comprovado cientificamente (Sousa, et al., 2020).

Em todo o mundo foi instalado estratégias que auxiliassem na contenção da transmissão da doença, dentre essas ações, destaca-se: o distanciamento social que consiste em evitar aglomerações de pessoas, proibindo por exemplo shows, eventos esportivos, aulas presenciais em escolas e universidades; e o isolamento social que consiste em as pessoas não saírem de suas casas com a finalidade de evitar a transmissão do vírus (Pereira, et al., 2020). A suspensão das atividades escolares, confinamento de crianças e adolescentes em suas casas durante a nova pandemia, acabou por afetar o desenvolvimento psicomotor e a saúde mental destes (Aydogdu, 2020).

Na infância e adolescência é o momento em que os circuitos neuronais dos jovens estão sofrendo constantes influências das experiências que a criança vivencia, gerando assim impactos no seu comportamento (Santos \& Celeri, 2018). Nessa perspectiva, o confinamento interfere nas potencialidades das crianças, provocando dificuldades para a aquisição de novas habilidades e capacidades, além de prejudicar as interações e relações interpessoais dos jovens que são importantes na fase da infância e adolescência (Aydogdu, 2020).

Nesse sentido, uma vez que as escolas consistem em um ambiente que permite a socialização das crianças fora do núcleo familiar, assim como seu desenvolvimento psicomotor e cognitivo, sendo ainda o local onde elas passam a maior parte do tempo durante o dia, logo com a paralisação das atividades escolares e a falta de interação com outras crianças, a falta destes têm o potencial de interferir na saúde mental dos estudantes (Sociedade Brasileira de Pediatria, 2020b; Melo, et al., 2020).

Além disso, as escolas além de atuarem como um espaço de aprendizagem do saber científicos, consistem também como importantes influenciadoras das habilidades sociais que uma criança necessita aprender (Macedo \& Medina, 2017), é através desses conhecimentos sociais que as crianças adquirem habilidades como uma boa comunicação, cooperação, engajamento em atividades, cortesia, relações harmoniosas com seus parentes, amigos e professores (Siqueira \& Freire, 2019).

O desenvolvimento dessas habilidades sociais permite que as crianças e adolescentes saibam como se relacionar e interagir em diferentes ambientes, permite que elas consigam lidar com os seus sentimentos, saibam como agir a uma agressão e consigam lidar com situações de estresse (Macedo \& Medina, 2017; Siqueira \& Freire, 2019). No entanto, todos esses fatores sofrem influência do modo como ocorreu o desenvolvimento das habilidades sociais na infância (Siqueira \& Freire, 2019), principalmente no contexto do isolamento social.

Assim, as mudanças nas vidas cotidianas dos jovens e o cenário atual da pandemia, no qual se perpetua o medo da infecção, o tédio, o estresse, o desamparo, a falta de contato com os amigos, a falta de atividades e a frustração, são fatores capazes de provocar consequências negativas para a saúde mental e o desenvolvimento infantil (Araújo, 2020). Logo, o presente 
estudo tem como objetivo discutir sobre o impacto biopsicossocial causado nas crianças devido o distanciamento e o isolamento social já relatados na literatura.

\section{Metodologia}

O presente estudo trata-se de uma pesquisa de natureza mista, ou seja, qualitativa e quantitativa, na qual torna-se importante a reflexão dos pesquisadores sobre os objetos em estudo e a análise de dados por meio de porcentagens e estatísticas. (Pereira, Shitsuka, Parreira, Shitsuka, 2018). Assim, Yin (2015) destaca que ao utilizar os métodos qualitativos e quantitativos juntos, é permitido uma maior compreensão a respeito do objeto em estudo. Nesse sentido, realizamos uma revisão sistemática da literatura, trazendo discussões e ponderações a respeito dos impactos psicossociais que podem acometer as crianças devido ao isolamento e distanciamento social em decorrência da pandemia da COVID-19.

Para isso, foi realizado consultas nos bancos de dados da SCIELO, MEDLINE e PUBMED, utilizando os descritores "children", "social isolation", "psychosocial" e "covid-19" os quais foram combinados pelo operador "and". Além disso, utilizou-se ainda os mesmos descritores em português: "crianças", "isolamento social", "psicossocial" e "covid-19". As buscas foram restringidas a artigos publicados durante o período de 2019 a 2020.

A partir do cruzamento dos descritores foram identificados o número de 64 artigos científicos, dentre os quais foram selecionados, num primeiro momento, 23 artigos de acordo com o objetivo desta revisão baseado na adequação do título. Posteriormente, realizou-se uma leitura minuciosa dos resumos dos artigos filtrados pelo título, obtendo uma amostra final de 15 publicações. Os trabalhos selecionados buscaram evidenciar como o isolamento social que foi colocado em prática em decorrência da atual pandemia pelo COVD-19, poderá provocar impactos psicossociais negativos na população infantil. Foram excluídos trabalhos de conclusão de curso, dissertações de mestrados, teses de doutorado, capítulos de livro e publicações que não contemplaram os objetivos desta revisão.

\section{Resultados}

Para essa revisão, foram selecionados um total final de 15 artigos após a leitura dos títulos e dos resumos. Os trabalhos selecionados buscaram evidenciar o impacto psicossocial provocado pelo isolamento social, devido a pandemia atual, nas crianças. O Quadro 1 apresenta os principais apontamentos de cada artigo selecionado.

Quadro 1. Síntese de indagações e desfechos principais identificados por autores.

\begin{tabular}{|c|c|c|c|}
\hline Ano & Autor & Principais questões identificadas & Desfecho principal \\
\hline 2020 & $\begin{array}{c}\text { Cao W., Fang, Z., Hou, G., } \\
\text { Han, M., Xu, X., Doung, J., } \\
\text { \& Zheng J. }\end{array}$ & $\begin{array}{c}\text { Aborda sobre a ansiedade entre } \\
\text { jovens provocada pela pandemia do } \\
\text { COVID-19. }\end{array}$ & $\begin{array}{c}\text { Crianças e adolescentes são um grupo } \\
\text { vulnerável a desenvolver } \\
\text { complicações psicológicas durante o } \\
\text { confinamento }\end{array}$ \\
\hline 2020 & $\begin{array}{c}\text { Tang, W., Hu, T., Hu, B., } \\
\text { Jin, C., Wang, G., Xie, C., } \\
\text { Chen, S. \& Xu, J. }\end{array}$ & $\begin{array}{c}\text { Destaca que a depressão e o } \\
\text { transtorno do estresse pós-traumático } \\
\text { são complicações que podem surgir } \\
\text { em crianças e adolescentes devido ao } \\
\text { isolamento social. }\end{array}$ & $\begin{array}{c}\text { As consequências psicológicas } \\
\text { derivadas do COVID-19 podem ser } \\
\text { graves para os estudantes }\end{array}$ \\
\hline 2020 & $\begin{array}{c}\text { Zhou, S., Zhang. L., Wang, } \\
\text { L., Guo, Z., Wang, J., Chen, } \\
\text { J., Liu, M., Chen, X. \& } \\
\text { Chen, J. }\end{array}$ & $\begin{array}{c}\text { Aborda sobre as consequências } \\
\text { psicológicas em crianças durante o } \\
\text { confinamento domiciliar. }\end{array}$ & $\begin{array}{c}\text { Há uma alta prevalência de problemas } \\
\text { psicológicos nos adolescentes devido } \\
\text { às medidas de contenção do COVID- } \\
19\end{array}$ \\
& \multicolumn{2}{|c}{}
\end{tabular}




\begin{tabular}{|c|c|c|c|}
\hline 2020 & $\begin{array}{c}\text { Oosterhoff, B. \& Palmer, C. } \\
\text { A. }\end{array}$ & $\begin{array}{l}\text { Mostra os impactos psicossociais } \\
\text { provocados pelo isolamento social } \\
\text { devido ao COVID-19. }\end{array}$ & $\begin{array}{c}\text { A pandemia apresentou um impacto } \\
\text { negativo no comportamento dos } \\
\text { estudantes }\end{array}$ \\
\hline 2020 & $\begin{array}{l}\text { Mechili, E.A., Saliaj, A., } \\
\text { Kamberi, F., Girvalaki, C., } \\
\text { Peto, E., Patelarou, A., } \\
\text { Bucaj, J. \& Patelarou, E. }\end{array}$ & $\begin{array}{l}\text { Evidencia as complicações sociais e } \\
\text { psicológicas que podem surgir com as } \\
\text { medidas de proteção contra a atual } \\
\text { pandemia. }\end{array}$ & $\begin{array}{l}\text { A saúde mental de estudantes } \\
\text { universitários foi negativamente } \\
\text { impactada pela quarentena }\end{array}$ \\
\hline 2020 & Ruíz-Roso, M., et al. & $\begin{array}{c}\text { Demonstra que as crianças estão } \\
\text { apresentando comportamentos } \\
\text { sedentários durante o confinamento } \\
\text { domiciliar. }\end{array}$ & $\begin{array}{l}\text { Houve uma maior prevalência de } \\
\text { inatividade das crianças e do } \\
\text { consumo de alimentos } \\
\text { ultraprocessados durante o } \\
\text { confinamento }\end{array}$ \\
\hline 2020 & $\begin{array}{l}\text { Türkoğlu, S., Uçar, H., } \\
\text { Çetin, F., Güler, H. \& } \\
\text { Tezcan, M. }\end{array}$ & $\begin{array}{c}\text { Aborda sobre os impactos do } \\
\text { isolamento social nas crianças que } \\
\text { apresentam autismo. }\end{array}$ & $\begin{array}{l}\text { As alterações no ciclo circadiano das } \\
\text { crianças e a desregulação no sono } \\
\text { provocados pelo confinamento } \\
\text { influenciam os sintomas do autismo }\end{array}$ \\
\hline 2020 & $\begin{array}{l}\text { Carroll, N., Sadowski A., } \\
\text { Laila, A., Hruska, V., } \\
\text { Nixon, M., Ma, D. W. L., \& } \\
\text { Haines J. }\end{array}$ & $\begin{array}{c}\text { Relata alterações nas atividades } \\
\text { físicas e na nutrição das crianças } \\
\text { durante o período de isolamento } \\
\text { social. }\end{array}$ & $\begin{array}{c}\text { Com as restrições do isolamento } \\
\text { social, houve um aumento no tempo } \\
\text { de tela e diminuição das atividades } \\
\text { físicas, além de mudanças nos hábitos } \\
\text { alimentares }\end{array}$ \\
\hline 2020 & $\begin{array}{c}\text { Sasaki, T., Niitsu, T., } \\
\text { Tachibana, M., Takahashi, } \\
\text { J. \& Iyo, M. }\end{array}$ & $\begin{array}{l}\text { Aborda sobre os impactos do } \\
\text { isolamento social nas crianças que } \\
\text { apresentam o Transtorno de Déficit } \\
\text { de Atenção e Hiperatividade. }\end{array}$ & $\begin{array}{c}\text { Piora dos sintomas de desatenção em } \\
\text { decorrência das medidas de } \\
\text { isolamento social }\end{array}$ \\
\hline 2020 & $\begin{array}{l}\text { R. Amorim,Catarino S., } \\
\text { Miragaia, P., Ferreras, C., } \\
\text { Viana, V., \& Guardiano M. }\end{array}$ & $\begin{array}{c}\text { Aborda sobre os impactos do } \\
\text { confinamento no comportamento das } \\
\text { crianças com autismo }\end{array}$ & $\begin{array}{l}\text { A pandemia do COVID-19 provocou } \\
\text { impactos psicológicos tanto nas } \\
\text { crianças com autismo, como nos seus } \\
\text { cuidadores }\end{array}$ \\
\hline 2020 & $\begin{array}{c}\text { Chen, S., Cheng, Z., \& Wu, } \\
\text { J. }\end{array}$ & $\begin{array}{c}\text { Destaca os fatores de risco que afetam } \\
\text { a saúde mental dos adolescentes } \\
\text { durante a pandemia do COVID-19 }\end{array}$ & $\begin{array}{c}\text { Durante a pandemia as crianças e } \\
\text { adolescentes ficam propícias à } \\
\text { ansiedade e depressão devido aos } \\
\text { vários fatores de risco }\end{array}$ \\
\hline 2020 & $\begin{array}{c}\text { Ezpeleta, L., Navarro, J. B., } \\
\text { Osa, N., Trepat, E., \& } \\
\text { Penelo, E. }\end{array}$ & $\begin{array}{c}\text { Relata sobre mudanças no } \\
\text { comportamento e nas relações sociais } \\
\text { de crianças e adolescentes durante o } \\
\text { confinamento }\end{array}$ & $\begin{array}{c}\text { Houve uma piora no relacionamento } \\
\text { dos adolescentes com os seus pais e } \\
\text { um alto nível de estresse }\end{array}$ \\
\hline 2020 & $\begin{array}{l}\text { Russell, B., Hutchison, M., } \\
\text { Tambling, R., Tomkunas, } \\
\text { A. J. \& Horton, A. }\end{array}$ & $\begin{array}{c}\text { Descreve sobre as dificuldades no } \\
\text { relacionamento de pais e filhos } \\
\text { durante o período de isolamento } \\
\text { social }\end{array}$ & $\begin{array}{l}\text { As medidas de isolamento em } \\
\text { detrimento dom COVID-19 } \\
\text { provocaram impactos negativos na } \\
\text { saúde mental de pais e filhos }\end{array}$ \\
\hline 2020 & $\begin{array}{l}\text { Manning, J., Billian J., } \\
\text { Matson, J., Allen, C. \& } \\
\text { Soares N. }\end{array}$ & $\begin{array}{c}\text { Aborda sobre as famílias de crianças } \\
\text { que apresentam autismo durante a } \\
\text { pandemia do COVID-19 }\end{array}$ & $\begin{array}{l}\text { Houve um aumento nos níveis de } \\
\text { estresse dos cuidadores e das crianças } \\
\text { com transtorno do espectro autista }\end{array}$ \\
\hline 2020 & $\begin{array}{l}\text { Zhang, J., Shuai, L., Yu, H., } \\
\text { Wang, Z., Qiu, M., Lu, L., } \\
\text { Cao, X., Xia, W., Wang, Y. } \\
\text { \& Chen, R. }\end{array}$ & $\begin{array}{c}\text { Destaca sobre os impactos do } \\
\text { isolamento social nas crianças que } \\
\text { apresentam déficit de atenção e } \\
\text { hiperatividade }\end{array}$ & $\begin{array}{l}\text { Durante a pandemia do COVID-19 as } \\
\text { crianças com TDAH apresentaram } \\
\text { uma piora nos sintomas de desatenção }\end{array}$ \\
\hline
\end{tabular}


Fonte: Autores (2020).

Dos 15 trabalhos analisados observou-se uma frequência de distúrbios psíquicos e alimentares, sendo citada emoções negativas por $40 \%$ dos autores, ansiedade em $33 \%$, depressão com $26 \%$; irritabilidade, sedentarismo e alteração do sono foi citado por $20 \%$ dos autores, estresse e alteração na alimentação foi citado por $13 \%$ e transtorno do estresse pós-traumático por $6 \%$ dos estudos, conforme representado no Gráfico 1.

Gráfico 1. Distribuição de distúrbios psíquicos citados por artigo.

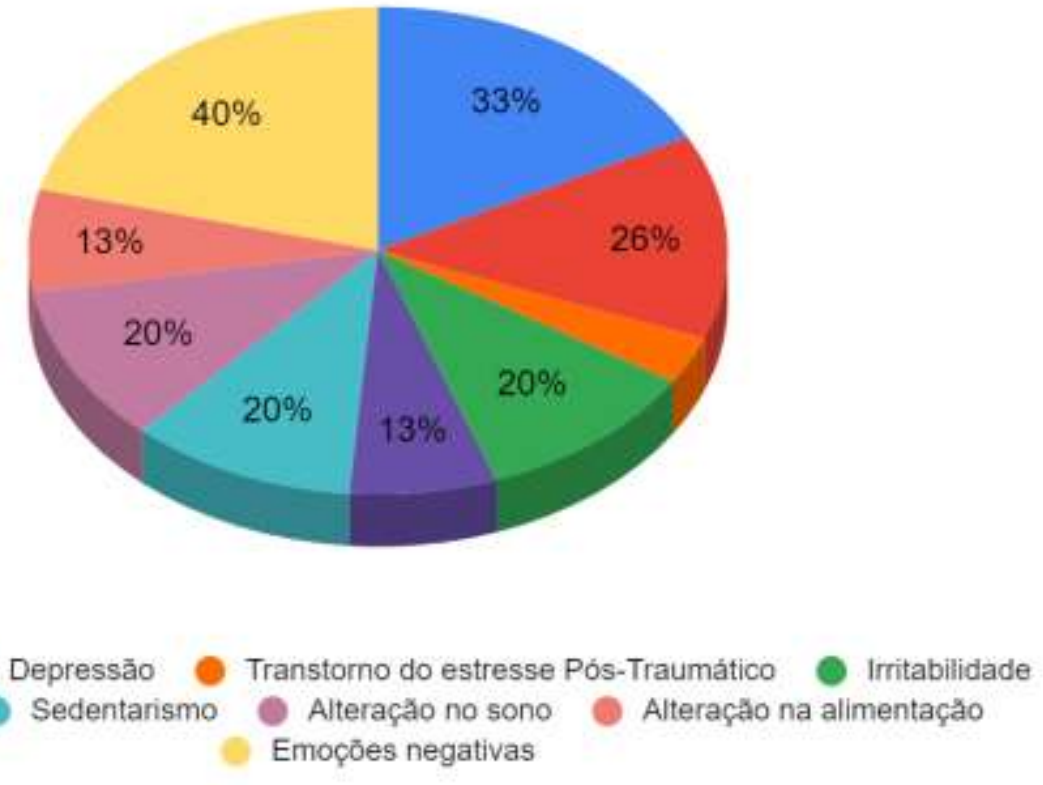

Fonte: Autores (2020).

\section{Discussão}

Um dos principais impactos, além do potencial de evolução a morte, a COVID-19 apresenta a capacidade de promover uma grande pressão psicológica nas pessoas (cao, et al., 2020). Nesse contexto, crianças e adolescentes mostram-se como um grupo vulnerável frente às complicações psicossociais do atual cenário, uma vez que eles não apresentam as mesmas capacidades de comunicação e entendimento da situação como os adultos (Cao, et al., 2020; Tang, et al., 2020) e com medidas de isolamento e distanciamento social adotadas para diminuir a disseminação do vírus, interrompeu-se o desenvolvimento das relações sociais das crianças e adolescentes (Mechili, et al., 2020; Tang, et al., 2020).

Além disso, o confinamento deixa as crianças durante um longo período em suas casas, ficando mais expostos a equipamento eletrônicos como televisões, computadores, celulares e tablets, logo, os jovens ficam expostos também à muitas notícias pelas mídias sociais, informações essas, geralmente, de cunho negativo sobre a própria doença e fatos diários que por vezes podem ser prejudiciais à saúde mental dos jovens (Mechili, et al., 2020). Esses elementos acabam influenciando de modo desfavorável no bem-estar físico e mental dessas crianças, as quais ficam propensas a desenvolver estresse crônico e agudo, preocupação com a saúde de seus familiares, ansiedade, depressão, distúrbios do sono e do apetite, irritabilidade, medo, insegurança e prejuízo nas interações sociais (Oosterhoff \& Palmer, 2020; Zhou, et al., 2020).

Nesse sentido, Cao et al. (2020) realizou estudo com a aplicação de questionários referentes a ansiedade a um grupo de 7.143 estudantes de Changzhi, na China, sendo demonstrado que dentre essa amostra, cerca de 24,9\% dos estudantes vivenciaram 
períodos de ansiedade decorrentes do surto de COVID-19, possibilitando os autores afirmar que a saúde dos estudantes universitários foi significamente afetada pela pandemia da COVID-19. Cao et al. (2020) destaca que a ansiedade desses estudantes pode ter sido afetada por fatores agravantes como a incerteza do futuro, e o aumento do distanciamento social, uma vez que a ausência de comunicação interpessoal agrava os transtornos de ansiedade, e ainda as mudanças no cotidiano devido ao isolamento social e a paralisação das atividades acadêmicas também estavam relacionadas com a ansiedade.

Esses achados de Cao et al. (2020) corroboram com o estudo de Chen, Cheng \& Wu (2020), o qual também evidenciou os fatores de risco que estavam mais relacionados com os casos de ansiedade durante a pandemia. Para isso, Chen, Cheng \& Wu (2020) aplicaram questionários online para 7.772 estudantes chineses, durante o período de isolamento social. Assim, ele mostrou que crianças e adolescentes que não apresentavam irmãos tinham um fator de risco a mais para desenvolver sintomas depressivos e de ansiedade, uma vez que a presença de um irmão reduziu o sentimento de solidão provocados nos jovens pelo confinamento, evidenciando que a ausência de comunicação interpessoal implica negativamente na saúde mental infantil (Chen, Cheng \& Wu, 2020; Cao et al., 2020).

Além dos sintomas de ansiedade, o confinamento domiciliar em decorrência da pandemia também está relacionado com quadros de depressão e transtorno do estresse pós-traumático (TEPT), nessa perspectiva, Tang et al. (2020) realizou um estudo com 2.485 estudantes, os quais foram submetidos a responderem questionários sobre o TEPT e sobre depressão, onde foi identificado que a prevalência de TEPT foi de $2,7 \%$ e $9 \%$ a de depressão, percentual referente ao total de participantes. Evidenciou-se ainda alterações no sono dos jovens, o medo de contrair a doença e viver em locais que apresentam um grande número de casos de pessoas infectadas pelo COVID-19, também foram fatores que proporcionaram sérias consequências psicológicas em adolescentes e crianças, sendo possível afirmar a existência da necessidade de intervenções psicológicas que visem trabalhar o sentimento do medo e melhoria da qualidade do sono das crianças (Tang, et al., 2020), fatos que corroboram com os achados de Cao et al. (2020) e os estudos de Zhou et al. (2020).

Zhou et al. (2020) evidenciou em suas pesquisas que há relação entre depressão e ansiedade nos jovens com a pandemia do COVID-19, em seu estudo aplicou-se questionários validados em 8.079 estudantes de ensino médio, os quais apresentavam entre 12 e 18 anos de idade, sendo identificado que cerca de 43.7\% apresentavam sintomas depressivos, 37,4\% sintomas de ansiedade e 31,3\% apresentavam uma combinação de sintomas depressivos e de ansiedade.

Nesse contexto, Zhou et al. (2020) observou que o isolamento social devido a pandemia provocou impactos psicológicos nos jovens, principalmente naqueles que estão cursando o último ano do ensino médio, uma vez que este é o período de modificações de nível escolar e onde há envolvido um contexto de grandes expectativas, por exemplo de provas importantes, vestibulares, findo do ensino médio. E o sentimento de incerteza provocado pelo atual cenário gerou maiores níveis de ansiedade nesses estudantes, com isso Zhou et al. (2020) sugere que atendimentos psicológicos no formato on-line seria uma medida de proteção a saúde mental dos mesmos.

Além da exposição ao fatores já mencionados, as crianças também ficam mais sujeitas a distúrbios alimentares e a um estilo de vida sedentário, nessa perspectiva Ruiz-Roso et al. (2020), realizaram pesquisa na qual utilizou-se a internet para aplicar o Questionário Internacional de Atividade Física e para realizar questionamentos referentes ao consumo de alimentos ultraprocessados durante o período do confinamento à 726 jovens, com idade entre 10 a 19 anos, o estudo identificou que 40,9\% dos jovens brasileiros já eram fisicamente inativos antes do isolamento, e que essa porcentagem aumentou para 93\% durante o período do confinamento, identificou-se ainda que os jovens passaram a consumir uma maior quantidade de alimentos ultraprocessados durante o isolamento social.

Ruiz-Roso et al. (2020) destaca que essa alteração alimentar, juntamente com a inatividade física, onde os jovens passam muito tempo assistindo TV e apresentam sono desregulado, são consequências do estresse e da ansiedade provocada pelo isolamento e que possuem forte potencial para o desenvolvimento da obesidade e comprometimento da saúde como um todo, 
corroboram com esses achados, nos estudos de Carroll et al. (2020) que realizaram estudo referente à saúde física e nutricional de pais e filhos durante a pandemia, para tal foi realizada em grupo de 254 famílias, dentre as quais foram obtidos dados sobre 310 crianças, com idade média de 6 anos, identificou-se que cerca de 52\% das crianças entrevistadas diminuíram suas atividades físicas e que $87 \%$ desses jovens aumentou o seu tempo de tela, o que promove o estilo de vida sedentário, que pode ser fator causador de futuras doenças crônicas.

Além disso, Carroll et al. (2020) observou que mais da metade dos participantes alterou sua rotina alimentar em detrimento das medidas de isolamento social devido a COVID-19. Dentre essas mudanças, houve um maior consumo de alimentos ultraprocessados, que apresentam alto teor de sódio, corantes, aditivos prejudiciais à saúde e pobre em nutrientes essenciais para o desenvolvimento pleno, possibilitando que crianças e jovens desenvolvem doenças como hipertensão arterial, diabetes e outras (Carroll, et al., 2020; Ruiz-Roso, et al., 2020), ainda é pontuado por Ruiz-Roso et al. (2020) que o consumo exacerbado de ultraprocessados também é incentivado pelo mercado das mídias sociais e televisivas das indústrias de alimentos, as quais estão sendo bastante assistidas pelas crianças nesse período em que elas não estão nas escolas.

Em sua pesquisa, Carroll et al. (2020) realizou ainda questionamentos relacionados ao nível de estresse das crianças durante a pandemia, e evidenciou que o comportamento e o humor infantil sofreram modificações durante esse período. Nesse sentido, os pais relataram que as crianças apresentavam mais irritabilidade, que elas se frustram mais facilmente e que apresentam um menor limiar de paciência. Os pais relataram ainda que as crianças ficaram muito tristes por não poderem ver seus amigos e familiares, e que eles não lidam bem com o tédio (Carroll, et al., 2020), o que é destacado pelos pesquisadores que esse mau comportamento dos jovens ocorre devido o estresse provocado pelas mudanças abruptas na rotina deles, em detrimento da necessidade do fechamento das escolas.

Corroborando com os achados de Carroll et al. (2020), os estudos de Ezpeleta et al. (2020) que também evidenciaram que houve mudanças no comportamento e nas relações das crianças durante o período de confinamento. Em sua pesquisa, Ezpeleta et al. (2020) aplicou questionários online para 226 pais, com filhos em idades média de 13 anos, e evidenciou que o relacionamento entre pais e filhos piorou, uma vez que os pais estavam sobrecarregados com seus trabalhos, discutiam com os filhos por causas dos deveres de casa, não conseguiam realizar atividades de lazer em família, e apresentavam altos níveis de estresse. Esses achados de Ezpeleta et al. (2020) também foram evidenciados pelas pesquisas de Russell et al. (2020).

Russell et al.(2020), por sua vez, aplicou questionários para 420 cuidadores de crianças dos 0 aos 18 anos de idade, onde foi possível identificar um aumento de conflitos nos relacionamentos entre pais e filhos, assim como evidenciado por Ezpeleta et al. (2020), e que essa piora possui correlação significativa com os sintomas de ansiedade e depressão, com a sobrecarga do cuidador e com o estresse infantil (Russell, et al., 2020). Desse modo, Russell et al. (2020) destaca que um bom relacionamento de pais e filhos, onde eles se sintam mais próximos, apresenta a capacidade de proporcionar bem-estar mental tanto para as crianças como para os seus cuidadores (Russell, et al., 2020).

Esses impactos biopsicossociais provocados pelas medidas de isolamento social também atingem grupos infantis mais vulneráveis, como os jovens que apresentam o transtorno do espectro autista (TEA) e as crianças com transtorno de déficit de atenção e hiperatividade (TDAH) (Manning, et al., 2020). Nesse contexto, Türkoğlu et al. (2020) desenvolveu uma pesquisa com 46 crianças que apresentam diagnóstico de TEA, as quais tinham entre 4 a 17 anos de idade, e aplicou os questionários "Autism Behavior Checklist", "Children's Sleep Habits Questionnaire" e o "Children's chronotype questionnaire" no início e no final do confinamento domiciliar.

Evidenciou que as crianças apresentaram maiores problemas no sono e ainda tiveram maiores pontuações no teste de cronotipo durante o período de confinamento em comparação com o período antes da pandemia, o teste de cronotipo busca avaliar o perfil do ciclo circadiano dos indivíduos (Türkoğlu, et al., 2020). Então, com esse teste Türkoğlu et al. (2020) conseguiu observar que as crianças com TEA apresentaram um cronotipo noturno durante o confinamento, o qual está relacionado com o 
comprometimento da capacidade de lidar com eventos adversos, como alteração da sua rotina, ficando mais suscetível a apresentar emoções negativas Além disso, Türkoğlu et al. (2020) relatou que a gravidade dos sintomas do autismo aumentaram à medida que o cronotipo das crianças ficaram mais noturnos e o sono delas mais desregulado, devido às medidas de isolamento social.

Amorim et al. (2020) também buscou evidenciar os impactos do confinamento na vida das crianças diagnosticadas com TEA, para tal foram aplicados questionários para 43 pais de crianças que apresentam TEA e para 56 pais de crianças que não apresentam essa condição, foi possível identificar que os pais de crianças com TEA relataram que houve muitas mudanças no comportamento de seus filhos, principalmente relacionados à ansiedade, irritabilidade, obsessão, hostilidade e impulsividade. Amorim et al. (2020) evidenciou ainda que as crianças com TEA apresentaram um impacto negativo da quarentena no gerenciamento das suas emoções, corroborando assim com os achados de Türkoğlu et al. (2020).

Sasaki et al. (2020) abordou sobre os impactos do isolamento sofrido pela população infantil mais vulnerável, voltado às crianças com TDAH, desse modo, foi realizado estudo com 15 crianças que apresentam o diagnóstico de TDAH, com média de 11 anos de idade, com aplicação aos pais e aos responsáveis legais pelas crianças o "ADHD Rating Scale", traduzido do inglês para Escala de Classificação do TDAH, o qual consiste em um conjunto de perguntas que visam verificar o comportamento das crianças. Desse modo, realizando a comparação das respostas dos testes antes e depois da quarentena, foi possível evidenciar que houve um aumento na pontuação total do ADHD Rating Scale, e ainda que o sintoma de desatenção apresentou uma piora significativa nas crianças com TDAH durante o período do confinamento (Sasaki, et al., 2020).

Sasaki et al. (2020) destaca que a piora no sintoma de desatenção pode estar relacionada com o trauma psicológico vivido pelas crianças durante a quarentena o que corrobora com os achados de Zhang et al. (2020), que realizou pesquisa aplicando questionários à 241 pais de crianças diagnosticadas com TDAH, as quais apresentavam de 6 a 15 anos de idade. Assim como Sasaki et al. (2020), Zhang et al. (2020) também destacou que o comportamento das crianças com TDAH apresentou uma piora significativa durante a pandemia quando em comparação com seu estado normal e ainda que esses sintomas do TDAH estavam interligados com o estado de humor negativos das crianças devido às medidas de confinamento.

\section{Conclusão}

O isolamento e o distanciamento social foram estratégias empregadas para a contenção da transmissão do novo coronavírus, provocando assim o fechamento de escolas, restaurantes e locais de interação social. Apesar dessas medidas serem utilizadas em prol da saúde da população com relação a pandemia, o bem-estar físico e mental das crianças sofreram impactos bastante negativos com o confinamento. Assim, com a quarentena houve um aumento de casos de ansiedade e depressão na população infantil e adolescente, os quais sofreram com sentimentos como solidão, incerteza, estresse, medo, frustração e irritabilidade. Além disso, as crianças ficaram mais propensas a um estilo de vida sedentário e a uma alimentação pouco nutritiva. Essas mudanças provocadas pela pandemia também atingiram grupos infantis mais vulneráveis, como os jovens que apresentam TEA e TDAH, as quais também sofreram com emoções negativas. Desse modo, nota-se que é imperativo a criação de medidas que visem auxiliar a saúde mental das crianças e dos adolescentes nesse período conturbado, oferecendo, por exemplo, sessões com psicólogos através de plataformas online.

Com base no exposto, torna-se importante o desenvolvimento de mais pesquisas que visem avaliar tanto os impactos à curto quanto à longo prazo provocados pelo isolamento social e pela pandemia do COVID-19 na população infantil, para que possa aprofundar mais sobre essa temática. 


\section{Referências}

Amorim, R., Catarino S., Miragaia, P., Ferreras, C., Viana, V., \& Guardiano M. (2020). The impact of COVID-19 on children with autism spectrum disorder. Revista de neurologia, 71(8), 285-291.

Araújo, J.N.G. (2020). Infância E Pandemia. Caderno De Administração, 28, 114-121.

Aydogdu, A.L.F. (2020). Children's mental health during the pandemic caused by the new coronavirus: integrative review. Journal Health NPEPS, 5(2), 1-17.

Brasil (2020). Ministério da Saúde. Secretaria de Vigilância em Saúde. Boletim Epidemiológico Especial - Doença pelo Coronavírus COVID-19. Brasília, 25(1).

Cao, W., Fang, Z., Hou, G., Han, M., Xu, X., Doung, J., \& Zheng J. (2020). The psychological impact of the COVID-19 epidemic on college students in China. Psychiatry research, 287.

Carroll, N., Sadowski A., Laila, A., Hruska, V., Nixon, M., Ma, D. W. L., \& Haines J. (2020). The Impact of COVID-19 on Health Behavior, Stress, Financial and Food Security among Middle to High Income Canadian Families with Young Children. Nutrients, 12(8), 2352.

Chen, S., Cheng, Z., \& Wu, J. (2020). Risk factors for adolescents' mental health during the COVID-19 pandemic: a comparison between Wuhan and other urban areas in China. Globalization and Health, 16(1), 1-11.

Ezpeleta, L., Navarro, J. B., Osa, N., Trepat, E., \& Penelo, E. (2020). Life Conditions during COVID-19 Lockdown and Mental Health in Spanish Adolescents. International journal of environmental research and public health, 17(19), 7327.

Freitas, B., Alves, M. \& Gaíva, M. (2020). Medidas de prevenção e controle de infecção neonatal por COVID-19: revisão de escopo. Revista Brasileira de Enfermagem, 73(2), 1-10.

Macedo, A. \& Medina, G. (2017). O que dizem os estudos brasileiros sobre o relacionamento interpessoal no ambiente escolar: Uma revisão de literatura. Revista PsicoFAE: Pluralidades em Saúde Mental, 6(1), 93-114.

Manning, J., Billian J., Matson, J., Allen, C. \& Soares N. (2020). Perceptions of Families of Individuals with Autism Spectrum Disorder during the COVID-19 Crisis. Journal of Autism and Developmental Disorders, 22, 1-9.

Mechili, E.A., Saliaj, A., Kamberi, F., Girvalaki, C., Peto, E., Patelarou, A., Bucaj, J. \& Patelarou, E. (2020). Is the mental health of young students and their family members affected during the quarantine period? Evidence from the COVID-19 pandemic in Albania. Journal of psychiatric and mental health nursing, 13(10), 1111

Melo, B., Pereira, D., Serpenoli, F., Kabad, J., Kadri, M., Souza, M., \& Rabelo, I. (2020). Saúde mental e atenção psicossocial na pandemia COVID-19: crianças na pandemia Covid-19. FIOCRUZ, 20.

Oosterhoff, B. \& Palmer, C. A. (2020). Psychological correlates of news monitoring, social distancing, disinfecting, and hoarding behaviors among US adolescents during the COVID-19 pandemic. PsyArXiv.

Pereira, A. S., Shitsuka, D. M., Parreira, F. J., \& Shitsuka, R. (2018). Metodologia da pesquisa científica: UFSM, NTE.

Pereira, M., Oliveira, L., Costa, C., Bezerra, C., Pereira, M., Santos, C. \& Dantas, E. (2020). A pandemia de COVID-19, o isolamento social, consequências na saúde mental e estratégias de enfrentamento: uma revisão integrativa. Research, Society and Development, 9 (7), 1-29.

Ruíz-Roso, M., et al. (2020). Changes of Physical Activity and Ultra-Processed Food Consumption in Adolescents from Different Countries during Covid-19 Pandemic: An Observational Study. Nutrients, 12(8), 2289.

Russell, B., Hutchison, M., Tambling, R., Tomkunas, A. J. \& Horton, A. (2020). Initial challenges of caregiving during COVID-19: Caregiver burden, mental health, and the parent-child relationship. Child Psychiatry \& Human Development, 51(5), 671-682.

Santos, R.; \& Celeri, E. (2018). Rastreamento de problemas de saúde mental em crianças pré-escolares no contexto da atenção básica a saúde. Revista Paulista de Pediatria, 36(1), 82-90.

Sasaki, T., Niitsu, T., Tachibana, M., Takahashi, J. \& Iyo, M. (2020). The Inattentiveness of Children with ADHD may Worsen During the COVID-19 Quarantine. Research Square.

Siqueira, A. \& Freire, C. (2019). A influência da tecnologia no desenvolvimento infantil. Revista FAROL, 8(8), 22-39.

Sociedade Brasileira de Pediatria (2020). Departamento Científico de Pneumologia. Nota de Alerta - COVID-19 em crianças: envolvimento respiratório. Sociedade Brasileira de Pediatria: São Paulo.

Sousa, M., Júnior, J., Reis, R., Andrade, B. \& Quintanilha, L. (2020). Patogênese e perspectivas de tratamento da Covid-19: uma revisão. Research, Society and Development, 9(7), 1-19.

Tang, W., Hu, T., Hu, B., Jin, C., Wang, G., Xie, C., Chen, S. \& Xu, J. (2020). Prevalence and correlates of PTSD and depressive symptoms one month after the outbreak of the COVID-19 epidemic in a sample of home-quarantined Chinese university students. Journal of affective disorders, $274,1-7$.

Türkoğlu, S., Uçar, H., Çetin, F., Güler, H. \& Tezcan, M. (2020). The relationship between chronotype, sleep, and autism symptom severity in children with ASD in COVID-19 home confinement period. Chronobiology International, 37(8), 1207-1213.

Yin, R. K. (2015). O Estudo de caso. Bookman. 
Research, Society and Development, v. 10, n. 2, e54210212286, 2021

(CC BY 4.0) | ISSN 2525-3409 | DOI: http://dx.doi.org/10.33448/rsd-v10i2.12286

Zhang, J., Shuai, L., Yu, H., Wang, Z., Qiu, M., Lu, L., Cao, X., Xia, W., Wang, Y. \& Chen, R. (2020). Acute stress, behavioural symptoms and mood states among school-age children with attention-deficit/hyperactive disorder during the COVID-19 outbreak. Asian journal of psychiatry, 51, 102077.

Zhou, S., Zhang. L., Wang, L., Guo, Z., Wang, J., Chen, J., Liu, M., Chen, X. \& Chen, J. (2020). Prevalence and socio-demographic correlates of psychological health problems in Chinese adolescents during the outbreak of COVID-19. European Child \& Adolescent Psychiatry, 29(6), 749-758. 